\title{
$\frac{N 65-21670}{\text { scentens }}$ \\ SEARCH FOR GAMMA-RAY POINT SOURCES
}

NASA +MX +55209

\author{
BY \\ C. E. FICHTEL \\ D. A. KNIFFEN
}

GPO PRICE $\$$

OTS PRICE(S) $\$$

Hard copy (HC) $2, y)$

Microfiche (MF)

.50

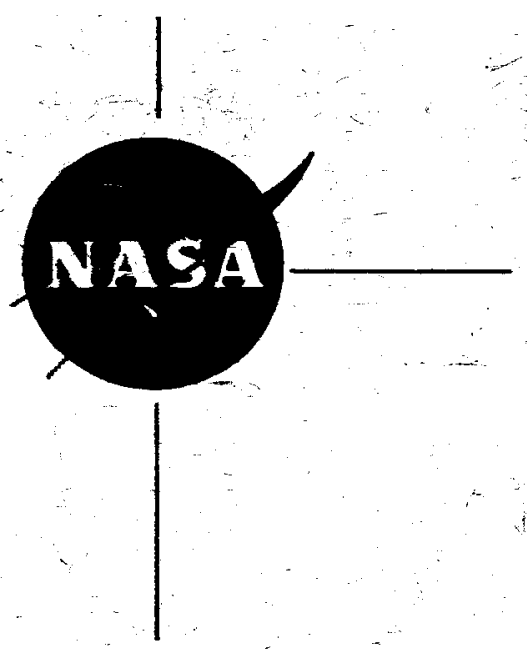

MARCH 1965

GODDARD SPACE FLIGHT CENTER

GREEMBELT, MARYLAND

Energetic Particles Preprint Series 
A SEARCH FOR GAMMA RAY POINT SOURCES

C. E. Fichtel and D. A. Kniffen

Goddard Space Flight Center

National Aeronautics and Space Administration

Greenbelt, Maryland

\section{0}

Abstract. A search for point sources of gamma rays has been made using oriented nuclear emulsions flown on high altitude balloons. The energy range examined was from 10 to $2 \times 10^{3} \mathrm{Mev}$, with the detection efficiency in the 10 to $50 \mathrm{Mev}$ region averaging about $10 \%$ which is almost a factor of ten greater than previous experiments with counter systems. No measurable flux of gamma radiation was observed for the regions of the sky examined, which included Cygnus $A$, the Spiral Arm In, Taurus A, Gemini, the Sun, and the Galactic Anticenter. A summary of the results of this and other experiments and theoretical considerations strongly suggest the need for further research in gamma ray astronomy.

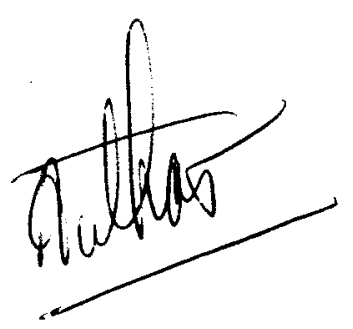




\section{$-2-$}

Introduction. The motivation for continued research in the field of gamma ray astronomy has been the desire to obtain information directly related to the high-energy nuclear processes which produce the great majority of the energy radiated from cosmic sources. Gamma rays have the advantage shared by charge particles of being closely related to many of the fundamental processes which are to be studied in astrophysics; yet they have the tremendous advantage over charged particles of not having their direction of motion changed by interplanetary magnetic fields, and hence the information related to the direction of the source position is not lost. Morrison [1958], who was one of the first to point out the possibility of obtaining information about highenergy cosmic processes through the direct mode of high-energy electromagnetic radiation, noted that both nuclear interactions of relativistic cosmic-ray protons with material and annihilation of nucleons and antinucleons were possible sources of mesons, which decay into gamma rays with a differential spectrum peaking at about $70 \mathrm{Mev}$. Several other processes, such as synchrotron radiation, inverse Compton collisions, and radioactive decay, produce photons whose average energy is 1 ower than $70 \mathrm{Mev}$.

Although it is expected that there also should be a general galactic background of gamma radiation, the experiment to be described here was primarily concerned with individual sources. Several other experiments [Braccesi and Ceccare11, 1960; Cline, 1961a; Frye and Reines, 1963; Kraushaar et a1., 19637 to look for point sources also have been 
undertaken during the last several years. None of these has found a measurable intensity coming from any of the potential point sources examined.

In an effort either to detect a source of gamma rays or to set upper limits, an experiment was undertaken to examine two of the more likely astrophysical source regions in the energy interval from 10 to $2000 \mathrm{Mev}$, with special emphasis on the energy region from 10 to $50 \mathrm{Mev}$, where the detection efficiency of most of the other experiments was very 1 ow. The detector used in this experiment was nuclear emulsion, which permits the unambiguous identification of each electron pair produced by a primary gamma ray and allows detailed measurements to be made. The principal disadvantage to nuclear emulsions is, of course, the fact that the data reduction is a long tedious process.

One of the bigger obstacles to gamma ray astronomy up to this time has been the high production rate of secondary gamma rays in the atmosphere. This problem can be solved to some degree by using very high altitude balloons to carry detectors above all but a few $\mathrm{g} / \mathrm{cm}^{2}$ of atmosphere and a sufficiently accurate pointing system. For this purpose, an orientation system was developed at the Goddard Space Flight Center to allow detector pointing angles accurate to within a cone with a $2^{\circ}$ full opening angle. This system has been flown twice on balloons to look primarily at Cygnus A and the Crab (Taurus A); however, the Spiral Arm In, the Galactic Anticenter, Gemini, and the Sun were also within the observed solid angle. The energy interval from 10 to $2 \times 10^{3} \mathrm{Mev}$ was examined. 
Apparatus and balloon flights. As suggested above, the principle problems related to the experimental platform to be flown on the balloon were: building a pointing system of sufficient accuracy, and measuring the actual orientation observed. It was determined that, for the proposed analysis and flight times, the average number of background pairs in the area to be scanned would be much less than 1 for a full cone opening angle of $2^{\circ}$. Therefore, the experimental design of the orientation system was aimed at maintaining a pointing accuracy of about $\pm 1^{\circ}$. Since the earth-sun line varies by only $1 / 4^{\circ}$ with respect to a galactic coordinate system during a balloon flight, the sun may be used as a "fixed" point in the galaxy for the accuracy desired. Proper control of the rotation of the detector about the earth-sun line during the flight can then keep the detector system at a constant orientation with respect to the galaxy.

In the first of the two experiments, use was made of a time resolution system wherein the individual nuclear emulsions were moved together mechanically during the ceiling portion of the balloon flight and were kept separated at other times. The purpose was to reduce the background which otherwise would be indistinguishable from the primary source. However, in the final analysis this feature was not used because the average number of background tracks of all types in the cone of uncertainty produced by errors in pointing accuracy was in fact less than 1 ; so there was little advantage in separating the types of background in terms of establishing point sources, and a considerable additional amount of work would have been involved in tracing the minimum electron tracks into an adjacent plate. 
The design of the platform for providing detector orientation with respect to the galaxy is described in detail elsewhere [Derdeyn and Kniffen, 1965]. The design was based on an earlier suntracker developed by Shechet [1960] and was modified to correct for the rotation of the earth and the balloon drift velocity during flight. Figure 1 shows the assembled system for the second of the two flights. In the earlier flight, the camera and emulsion package were placed at opposite ends of a shaft whose center was attached to the rotating cylinder. The shaft lay in the plane perpendicular to the sun, and the camera and detector rotated about the earth-sun vector as required. This configuration was designed to insure that portions of the gondola frame would not block the detector from the potential sources during portions of the flight. Different arrangements for the two flights are required because of the different positions of the celestial objects with respect to the gondola frame.

The sunseeker design depended primarily on two Hof fman type-220C photocells separated by a shade [see Shechet, 1960], designed so that a drift from proper orientation would cause an imbalance of sunlight between the two cells. The output of the cells provided an input signal to a Barber Coleman Micropositioner Relay in such a manner that the imbalance of light actuated the relay which, in turn, applied power to a reversible orientation motor and thus corrected the pointing error. Three axes of movement were built into the gondola, with the photocells providing azimuthal and elevation orientation with respect to the sun and therefore two of the axes of orientation. The effect of earth motion 
and balloon drift is to cause a celestial source to rotate about the earth-sun line at a nonuniform rate. From Figure 2 it can be shown that the angle of rotation required is given by

$$
\beta=\arctan \left[\frac{\cos \theta_{\mathrm{v}} \sin \left(\phi_{\mathrm{v}}-\phi_{\mathrm{S}}\right)}{\sin \theta_{\mathrm{v}} \cos \theta_{\mathrm{S}}-\cos \theta_{\mathrm{v}} \cos \left(\phi_{\mathrm{v}}-\phi_{\mathrm{S}}\right) \sin \theta_{\mathrm{s}}}\right]
$$

where

$$
\begin{aligned}
& \theta_{\mathrm{v}}=\text { latitude of the balloon } \\
& \theta_{\mathrm{S}}=\text { declination angle of the sun } \\
& \phi_{\mathrm{v}}=\text { right ascension angle of the vertical } \\
& \phi_{\mathrm{S}}=\text { right ascension angle of the sun }
\end{aligned}
$$

In Figure 2, $\beta$ is measured from the perpendicular projection of the north vector onto the plane perpendicular to the sun. Thus $\beta=0$ represents true noon at the longitude of the balloon.

It can be seen that $\beta$ is a function of time during the flight, since $\phi_{V}-\phi_{S}$ is a function of time. To provide this correction, a punched-tape programmer which made corrections in steps of 10 minutes of arc, as required by equation 1 , was developed. The tape was then punched as late as possible prior to launch, so that maximum information on the effect of high-altitude winds on $\not_{v}-\phi_{s}$ could be fed into the punched-tape program.

The accuracy of the achieved orientation was measured in two ways. First, photographs were taken of the sun at regular intervals during the flight by a motor-driven Nikon F camera with a $500 \mathrm{~mm} 1 \mathrm{ens}$. The lens 
opening appears in Figure 1 and is rigidly connected to the central shaft which pointed to the sun throughout oriented flight. Figure 3 shows the results of the photographs taken during the Churchill flight, with each data point representing the average sun position in the field of view for ten exposures (30 minutes). The figure shows that throughout the early part of the flight excellent orientation was obtained, becoming relatively poorer toward the end of the flight but stil1 within about 1.5 degrees of the earlier points.

The second check was made to determine that the programmer orientation provided the proper rotation $\beta$ about the earth-sun axis. This was accomplished by a brush, commutator arrangement along the central rotating cylinder. The commutator had alternate strips of grounded conductor and insulator so that, for every $1.5^{\circ}$ of rotation in $\beta$, the brush changed from a grounded to an open circuit and back. The signal caused a 1000-cps frequency shift in the CW tracking beacon, which was easily detected at a ground station and was checked against the expected rate of rotation for proper orientation.

The observed errors in orientation were folded into the conversion from the angles in the nuclear emulsions to celestial coordinates and are reflected in Figures $4(\mathrm{a})$ and $4(\mathrm{~b})$ by the solid angles subtended by the potential sources during flight.

The times and places selected for balloon launching were chosen in an attempt to minimize the amount of atmosphere between the source and the detector and to avoid horizon effects. Hence, a latitude was chosen 
to correspond closely to the declination angle of the primary source and a time of the year such that the right ascension angle of the source is near that of the sun, so that the source is overhead (or nearly so) midway during the flight. Table 1 shows the right ascension (R.A.) and declination angle (D.A.) of the sun and the sources for the two flights. The Texas site was chosen for the primary purpose of looking at Cygnus $A$, since it was the most southerly launch site which was easily available. Neepawa in southern Manitoba was chosen for a June 1963 flight to observe the Crab Nebula, but damage to the orientation equipment during the balloon launches required the alternate choice of Fort Churchill in August 1963. Table 2 gives some of the essential flight parameters. The relatively large solid angle, subtended by the sources during the Texas flight, was due to the wind predictions prior to the flight being grossly in error; and hence there were significant errors in the $\phi_{\mathrm{V}}-\phi_{\mathrm{S}}$ value and consequently in $\beta$, as shown in Table 2 and Figure $4(a)$.

Data analysis. The gamma rays were detected in the emulsions by observing electron-positron pairs produced in the emulsion itself. Herein lies one of the major advantages of emulsion over the scintillation-Cerenkov telescope detectors. For the latter, conversion material is required between the gammas and the detector which has the disadvantage that Compton scattering before pair formation, and electron coulomb scattering afterward, reduces severely the detection efficiency at low energies (10 - $50 \mathrm{Mev)}$ as depicted in Figure 5. Thus, the emulsion detector 
serves to extend the observable gamma ray energies downward to about $10 \mathrm{Mev}$. It should be noted that, in this figure, emulsion detector efficiency is based on pair production probabilities at the "scan line" based on $3.86 \mathrm{~cm}$ of emulsion conversion length, while the counter data are based on actual calibrations [C1ine, 1961b].

Thirty percent of the scanned area of each scanner was rescanned by a different scanner to obtain scanning efficiencies. Because of the tedious and difficult task of looking for minimum ionizing tracks, the gamma ray detection efficiencies always are 1 ow compared with those normally obtained in emulsion work; however, insofar as the efficiencies can be determined reliably, this is no serious difficulty except for the fact that it causes a significant increase in the scanning time-already quite lengthy for an area scan-to obtain the same statistics.

The efficiencies were calculated by the method of Lim et al. [1960], which can in general lead to a small overestimate of the scanning efficiency [Waddington, 1961]. However, the most serious variation in scanning efficiency occurs in going to large emulsion dip angles. This uncertainty has been largely avoided by limiting the analysis to dip angles of less than $30^{\circ}$ in the unprocessed emulsions. Cross correlations also indicated no significant variation in efficiency with the opening angle of the pair in the region of values of interest. The error in determining the efficiency, because of the above considerations, is probably less than 5 percent, and hence is not significant in an 
experiment where the principal concern is establishing order-of-magnitude upper limits.

In addition, about 20 percent of the accepted tracks were further examined to determine the opening angle of the electron pair in order to obtain an indication of the shape of the energy spectrum of the observed gamma rays. This, however, represents only an atmospheric background spectrum, since no observable source flux was detected.

Results. A plot of the coordinates of all gamma rays found in the two flights appears in Figures $4(\mathrm{a})$ and $4(\mathrm{~b})$. The area of the figures representing the various sources represents approximately the angle subtended by the source at the emulsion detector during the period of oriented flight. The solid angle subtended includes errors due to pointing inaccuracies, uncertainties in measurement, and determination of the space angles. The larger solid angle for the Texas flight represents the error introduced because of the large errors in predicted wind velocities.

In part of the Fort Churchill scan, the solid-angle interval was limited to the area outlined by the dotted lines; and therefore there is a relative dearth of points outside these dotted lines.

Table 3 presents the upper limits to gamma rays from the various sources in three different energy intervals. The calculation was made by adding 1 to the number of gamma rays observed in the subtended solid angle following the method of Kraushaar and Clark [1963]. The limits 
include extrapolation to the top of the atmosphere and corrections for scanning and pair production efficiencies. The division into energy intervals is made because of the lack of any information on the shape of the source spectrum since, of course, pair production efficiencies and loss rates in the intervening material are energy-dependent.

It should be pointed out that the upper limit to gamma rays with energies greater than 10 Mev cannot be obtained simply by adding the upper limits in the three energy intervals. Such a 1 imit would be too large and would correspond roughly to adding 3 to the number of gamma rays observed in the appropriate solid angle. The upper limit on all gamma rays with energies greater than 10 Mev probably would correspond more closely to the limit in the 50 to $200 \mathrm{Mev}$ interval-depending, of course, on the shape of the source spectrum.

The new data reported here in the 10 to $50 \mathrm{Mev}$ interval set important limits on the bremsstrahlung, synchrotron radiation, and inverse Compton scattering processes which could make contributions in this region. The expected photon spectrum in each case is directly dependent on the parent electron spectrum and, particularly, the maximum electron energy. If a particular type of mechanism is assumed to be operative, calculations based on rather uncertain parameters-and in some cases on visual observations and measured radiation at other frequencies-can be performed to estimate the gamma ray flux and energy spectrum. The estimates for the high-energy gamma radiation are very uncertain, not only because of the limited knowledge of the parameters involved but a1so because of the 
fact that the extrapolation to the gamma ray frequencies must be made over a large frequency interval-an interval in which there is a rather high probability of the spectral slope steepening because of the possible sparsity of very high energy electrons at the source. Therefore, any calculation of gamma ray intensity based on these assumptions must be viewed with some reservations. However, since this approach appears to be the best possible one at this time, a few estimates were made.

For the case of Cygnus A, if a spectral shape of the form $I=A \nu^{-b}$ is assumed, and $f 1$ ux data in the radio [Maltby et a1., 1963; Shkolvsky, 1960] and optical [Baade and Minkowski, 1954] regions are used for normalization, $b$ is found to be 0.88 [Maltby et al., 19637; and the flux in the energy region from 10 to $50 \mathrm{Mev}$ is calculated to be $0.7 \times 10^{-5} \mathrm{~cm}^{-2} \mathrm{sec}^{-1}$. The optical data include a correction for absorption in interstellar matter, although this correction is very uncertain. In a similar manner, the flux in the 50 to $200 \mathrm{Mev}$ region would be about the same. If, on the other hand, one assumes that the recent Cygnus XR-1, $\mathrm{x}$-ray source detected by Bowyer et a1. [1965] is the same as the radio source [Shklovsky, 1960], one obtains for a flux of $1.5 \times 10^{-8}$ ergs $\mathrm{cm}^{-2} \mathrm{sec}^{-1}$ in the 1.5 to 8 angstrom region a value for $A$ of $2.0 \times 10^{-13}$ ergs $\mathrm{cm}^{-2} \mathrm{sec}^{-2} \mathrm{c} / \mathrm{s}^{-1}$ and a value for $b$ of 0.72 . Such an assumption about the source of the two observations may not be justified since the celestial coordinates do not seem to coincide; however, the interesting result is that the spectral index $b$ is in reasonably good agreement with the index obtained in the radio region [Ma1tby et a1., 1963]. Such an assumption then gives a gamma ray flux 
in the 10 to $50 \mathrm{Mev}$ region of $7.5 \times 10^{-3} \mathrm{~cm}^{-2} \mathrm{sec}^{-1}$, almost a factor of 3 higher than the upper limit set by this experiment.

A similar calculation for the Crab Nebula (Taurus A) gives a gamma ray flux for the same energy interval of $3 \times 10^{-6} \mathrm{~cm}^{-2} \mathrm{sec}^{-1}$, well below the observed upper limit. Other sources investigated in this experiment yield extrapolated fluxes well below observable limits. Although the objectives of this experiment did not include measurements on the general background flux, a few remarks are probably worthwhile. Firstly, the background intensity observed is the result of electron pairs formed before the balloon flight, during ascent and descent, and in flight. The actual number observed agreed with the number expected to within the uncertainties involved in estimating the background, which are probably about a factor of 2. In calculating the expected background, use was made of the earlier balloon ascent data of Cline [1961a].

Concluding remarks. The negative results of this and other experiments [Braccesi and Ceccarelli, 1960; Cline, 1961a; Frye and Reines, 1963; Kraushaar et al., 1963] set important limits to the intensity of various possible gamma ray sources. In this experiment, in addition to confirming the absence of a detectable flux in the energy region above $50 \mathrm{Mev}$ at the intensity levels discussed, new limits were set in the energy region from 10 to $50 \mathrm{Mev}$ for several sources. Several authors have now estimated the source strengths of many possible sources-using models in which several physical processes are assumed, including synchrotron radiation, neutral pion decay, inverse Compton collisions, bremsstrahlung, radioactive decay, electron 
positron annihilation, and others [Savedoff, 1959; Milford and Shen, 1962; Pollack and Fazio, 1963; Dolan and Fazio, 1964]. In general, such estimates are extremely speculative; but, excluding synchrotron radiation and the newly postulated quasi-stellar sources, there seem to be no source mechanisms which will produce fluxes at the earth higher than about $10^{-6}$ to $10^{-8}$ photon $\mathrm{cm}^{-2} \mathrm{sec}^{-1}$ above $10 \mathrm{Mev}$. Synchrotron radiation or quasi-stellar sources may produce higher fluxes, as observed at the earth.

It is now clear that new experiments, which can detect much weaker sources than the limits which have been set, must be performed. Further, the existing experimental limits and present source strength estimates strongly suggest that it will be necessary to place these experiments above the earth's atmosphere in satellites in order to avoid the background produced in the overlying atmosphere on a balloon flight from obscuring the primary flux.

Acknowledgment. The authors are indebted to Mr. S.M. Derdeyn for his contribution to the development of the orientation system and his outstanding effort in the preparation of the payload for flight in the field. 
Maltby, P., Matthews, T. A., and Moffet, A. T., Brightness distribution in discrete radio sources. IV-A discussion of 24 identified sources, Ap. J., 137, 153-163, 1963.

Milford, S. W., and Shen, S. P., Cosmic-ray and diffuse-matter information from extraterrestrial gamma rays, I1 Nuovo Cimento, 23, 77-87, 1962 .

Morrison, P., On gamma ray astronomy, I1 Nuovo Cimento, 2, 858-864, 1958. Pollack, J. B., and Fazio, G. G., Production of $\pi$ mesons and gamma radiation in the galaxy by cosmic rays, Phys. Rev., 131, 2684-2691, 1963.

Savedoff, M. P., The crab and Cygnus A as gamma ray sources, I1 Nuovo Cimento, $\underline{7}, 1584-1590,1959$.

Shechet, M. L., Lightweight suntracker for balloon applications, Review of Scientific Instruments, $31,546,1960$.

Shklovsky, I. S., Cosmic Radio Waves, Harvard University Press, Cambridge, 1960.

Waddington, C. J., A remark on the detection of events in nuclear emulsions, Il Nuovo Cimento, 19 (supp1.), $37-40,1961$. 


\section{REFERENCES}

Baade, W., and Minkowski, R., Identification of the radio sources in Cassiopeia, Cygnus and Puppis A, Ap. J., 119, 206-214, 1954. Baade, W., and Minkowski, R., On the identification of radio sources, Ap. J., 119, 215-231, 1954 .

Bowyer, S., Byram, E. T., Chubb, T. A., and Friedman, H., Cosmic X-ray sources, Science, 147, 394-398, 1965.

Braccesi, A., and Ceccarel1i, M., Search for gamma-radiation from the Cygnus A radiosource, I1 Nuovo Cimento, 17, 691-694, 1960.

Cline, T. L., Search for high energy cosmic rays, Phys. Rev. Letters, ㄱ, $109-112,1961 \mathrm{a}$.

Cline, T. L., An investigation in high energy gamma-rays, $\mathrm{Ph} . \mathrm{D}$. Thesis, Mass. Inst. of Technology, 1961b.

Derdeyn, S. M., and Kniffen, D. A., A system for orienting balloon payloads in space, to be published, 1965.

Dolan, J.F., and Fazio, G. G., The gamma-ray spectrum of the sun, to be published in the Reviews of Geophysics, 1965.

Frye, G. M., Jr., and Reines, F., Search for primary cosmic gamma-rays, Bul1. Am. Phys. Soc., 8, 292, April 1963.

Kraushaar, W. L., Clark, G. W., Agagino, M., Garmire, G., Helmken, H., and Nigbie, P., Cosmic gamma ray results from Explorer XI, International Conference on Cosmic Rays, Jaipur, December 1963.

Lim, Y. K., Laby, J. E., and Hopper, V. D., Efficiency in area scanning for events in nuclear emulsions, Il Nuovo Cimento, 15 (suppl.), 382-386, 1960 . 
Table 2 Flight Parameters

\begin{tabular}{lcc} 
Parameter & $\begin{array}{c}\text { Texas } \\
\text { Flight }\end{array}$ & $\begin{array}{c}\text { Churchill } \\
\text { Flight }\end{array}$ \\
\hline Date & Jan. 21,1963 & Aug. 3,1963 \\
Latitude & $33.0 \% \mathrm{~W}$ & $58.6^{\circ} \mathrm{N}$ \\
Longitude & $98^{\circ}-105^{\circ} \mathrm{W}$ & $94^{\circ}-102^{\circ} \mathrm{W}$ \\
$\begin{array}{l}\text { Duration } \\
\begin{array}{l}\text { Approximate } \\
\text { Altitude }\end{array}\end{array}$ & 3 hours & 9.6 hours \\
$\begin{array}{l}\text { Range of error } \\
\text { in } \beta\end{array}$ & $4.9 \mathrm{~g} / \mathrm{cm}^{2}$ & $4.5 \mathrm{~g} / \mathrm{cm}^{2}$ \\
\hline
\end{tabular}


Table 1 Celestial Coordinates

\begin{tabular}{lcc}
\hline Source & $\begin{array}{r}\text { R.A. ( } \phi) \\
\text { (degrees) }\end{array}$ & $\begin{array}{c}\text { D.A. ( }) \\
\text { (degrees) }\end{array}$ \\
\hline Sun (Jan. 21, 1963) & 302.0 & -20.1 \\
Cygnus A & 300.0 & +40.5 \\
Spiral Arm In & 311.0 & +45.0 \\
\hline Sun (Aug. 3, 1963) & 133.0 & +17.8 \\
Taurus A & 83.30 & +22.0 \\
Gemini & 93.30 & +22.6 \\
Galactic Anticenter & 75.00 & +30.0 \\
\hline
\end{tabular}


Table 3 Flux Limits $\left(\mathrm{cm}^{-2} \mathrm{sec}^{-1}\right)$

\begin{tabular}{lccc}
\hline \multirow{2}{*}{ Source } & \multicolumn{3}{c}{ Energy Interval } \\
\cline { 2 - 4 } & $10-50 \mathrm{Mev}$ & $50-200 \mathrm{Mev}$ & $>200 \mathrm{Mev}$ \\
\hline Cygnus A & $2.6 \times 10^{-3}$ & $1.8 \times 10^{-3}$ & $1.6 \times 10^{-3}$ \\
Spiral Arm In & $8.0 \times 10^{-3}$ & $5.3 \times 10^{-3}$ & $5.0 \times 10^{-3}$ \\
Taurus A & $4.2 \times 10^{-3}$ & $2.9 \times 10^{-3}$ & $2.8 \times 10^{-3}$ \\
Gemini & $2.2 \times 10^{-3}$ & $1.5 \times 10^{-3}$ & $1.4 \times 10^{-3}$ \\
Sun & $1.5 \times 10^{-3}$ & $1.0 \times 10^{-3}$ & $0.9 \times 10^{-3}$ \\
Galactic Anticenter & $5.1 \times 10^{-3}$ & $3.5 \times 10^{-3}$ & $3.4 \times 10^{-3}$ \\
\hline
\end{tabular}




\section{FIGURE CAPTIONS}

Fig. 1. The gondola flown on the Churchill flight. (The Texas flight gondola was similar.)

Fig. 2. A three-dimensional display of the geometry involved in determining the rotation required about the earth-sun axis.

Fig. 3. A graphic representation of pictures taken of the sun on the Churchill flight. (Each data point represents the mean position of the center of the sun's image for ten exposures -30 minutes of flight.)

Fig. 4.

(a) Texas flight

(b) Churchi11 flight

A plot of the direction of arrival of the detected electron pairs vs. emulsion coordinates and celestial coordinates.

Fig. 5. A comparison of detection efficiences vs. energy for nuclear emulsion and scintillator-cerenkov detector. 

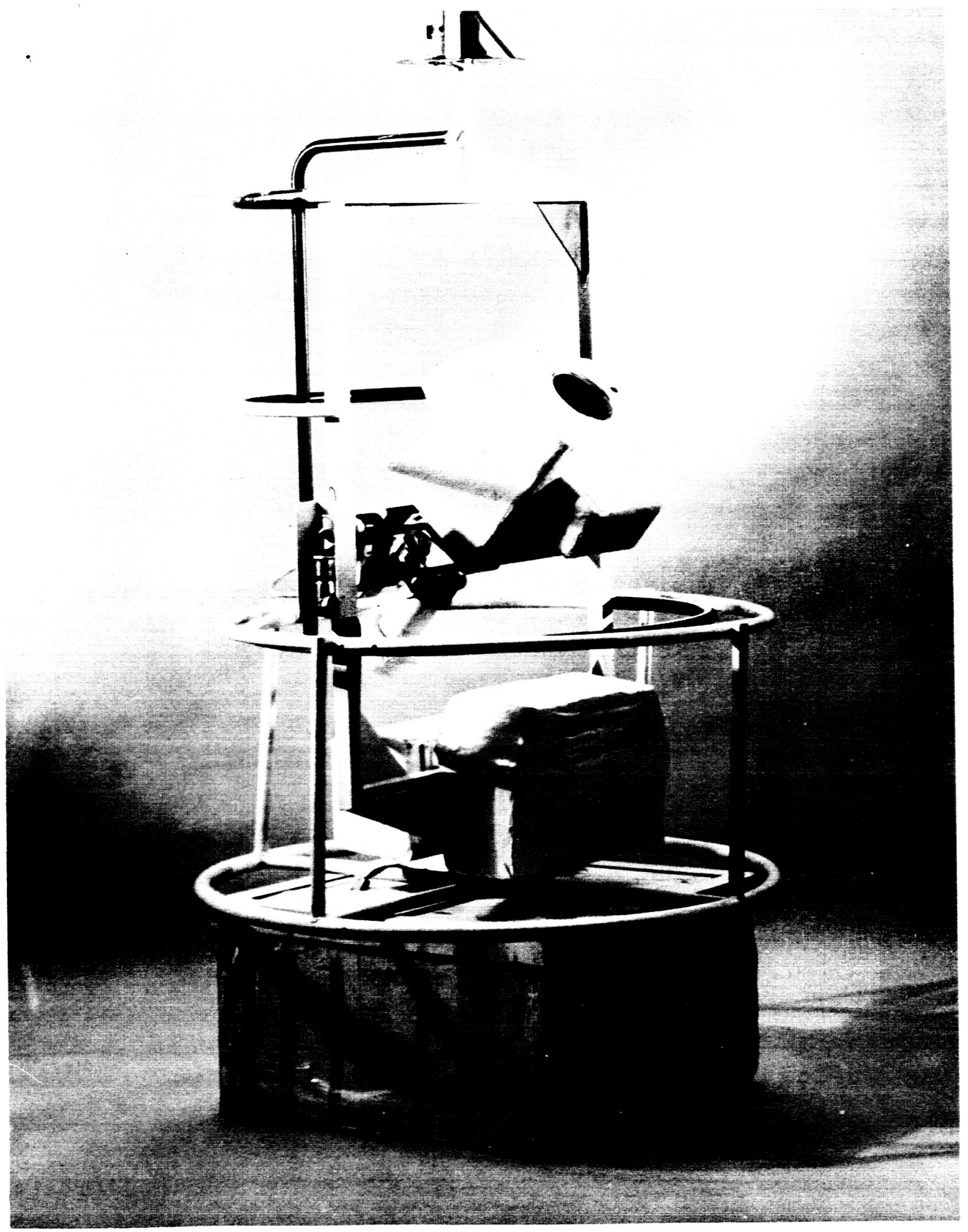


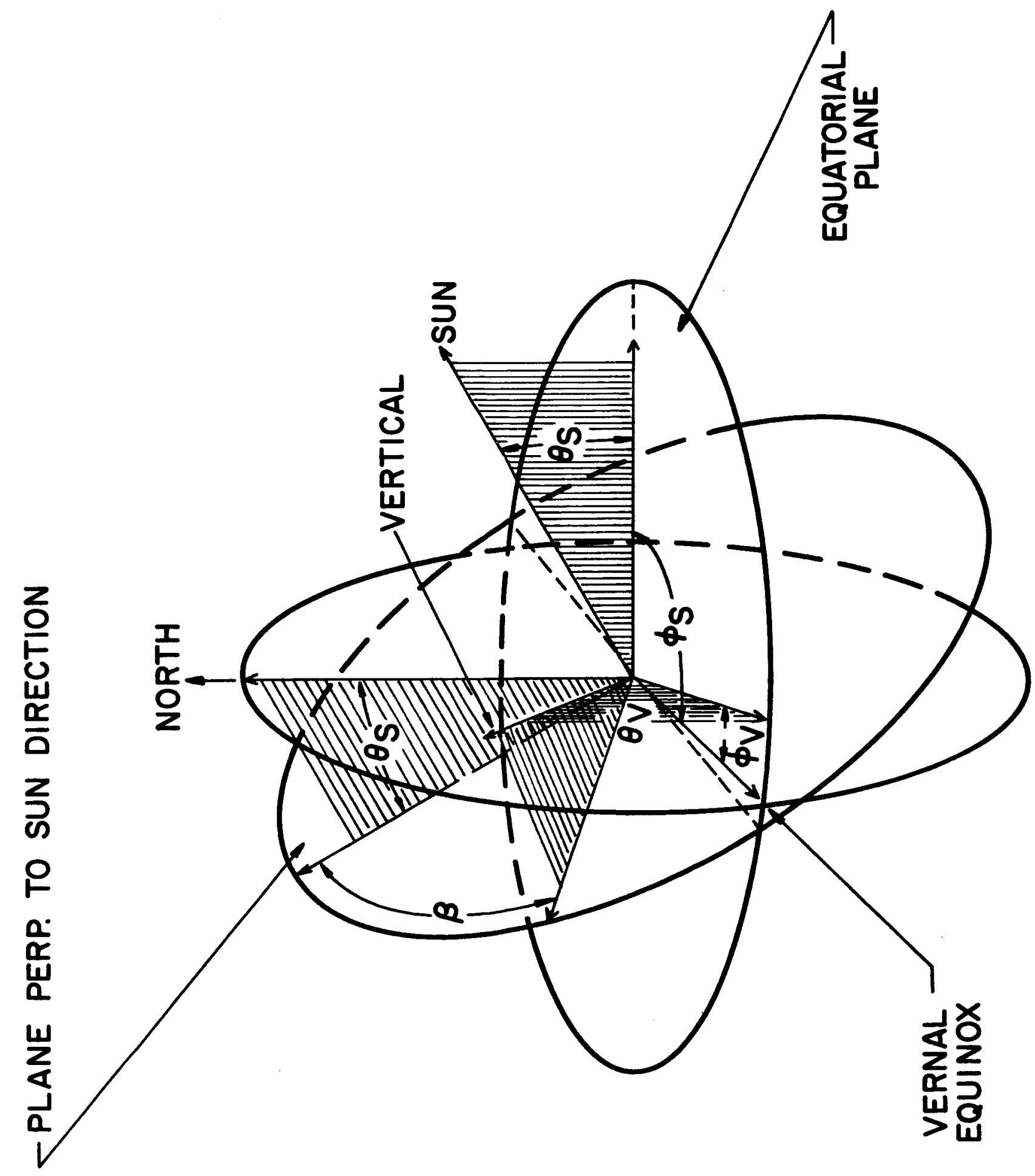




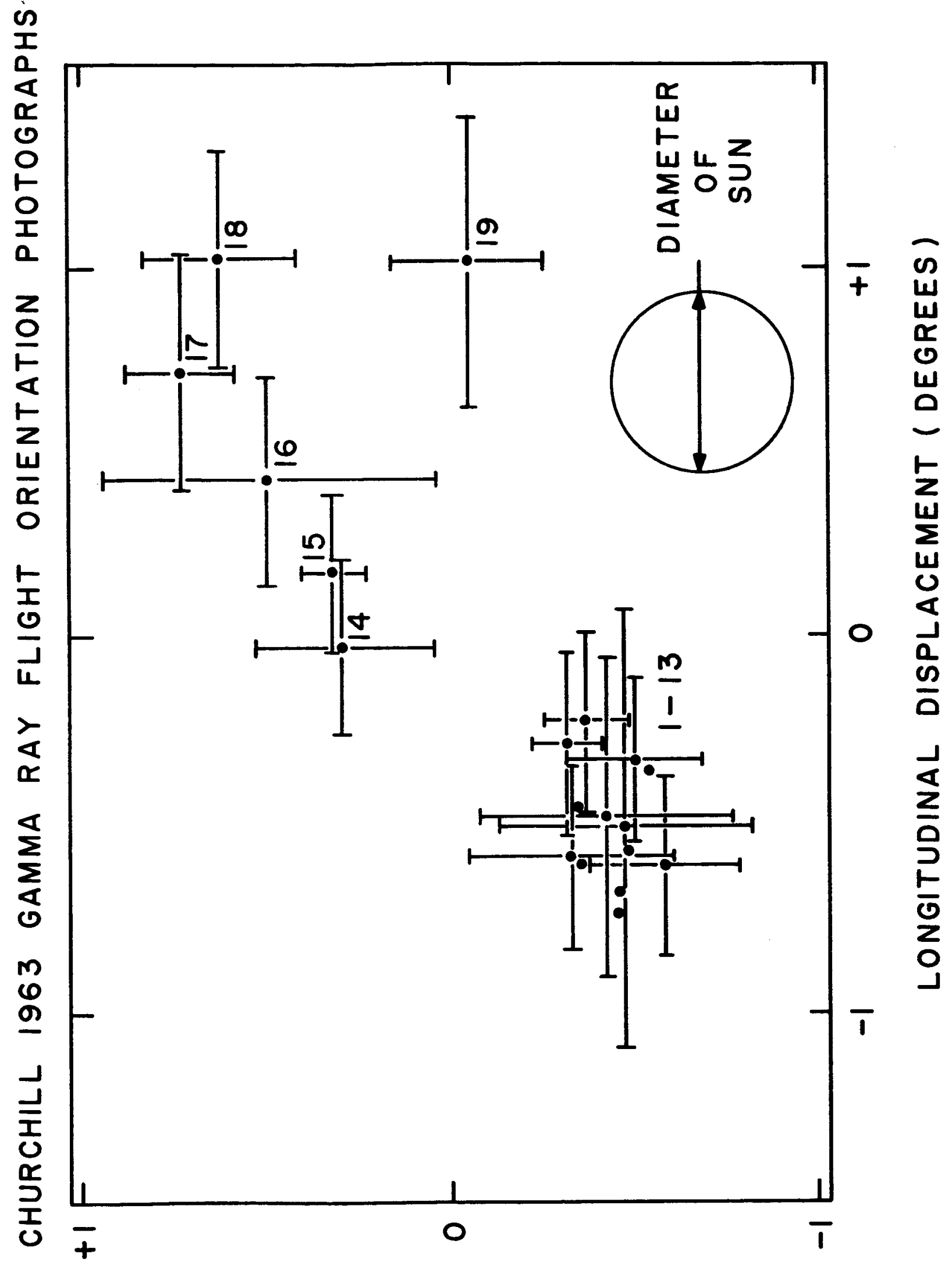

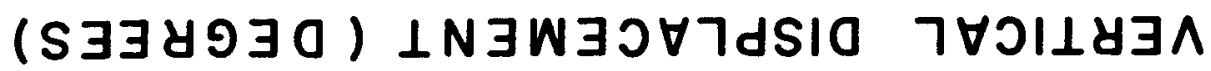

FIGURE 3 
(

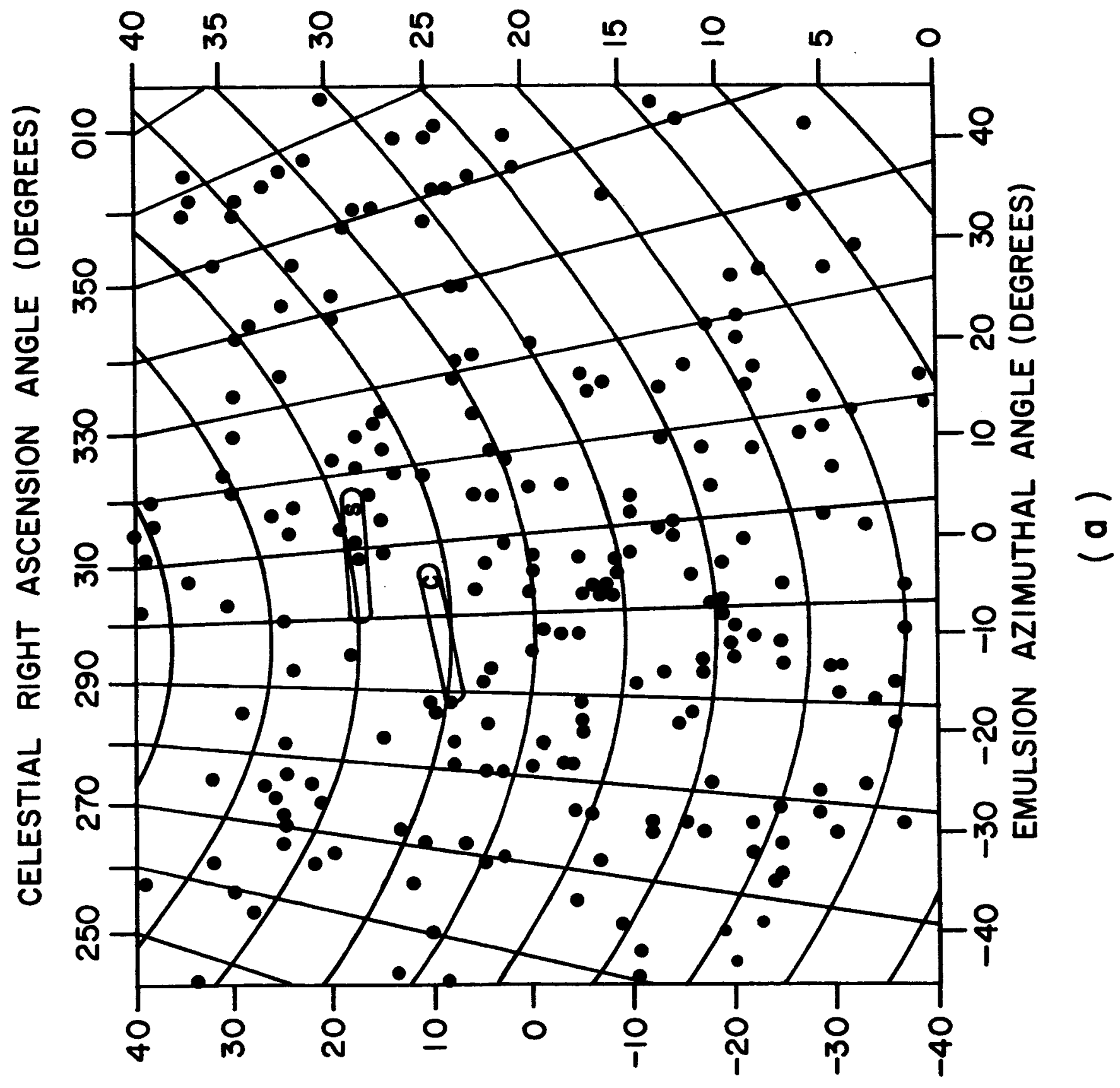

(SNOצJIW) dIO NOISרกW 


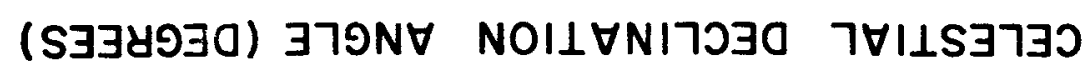

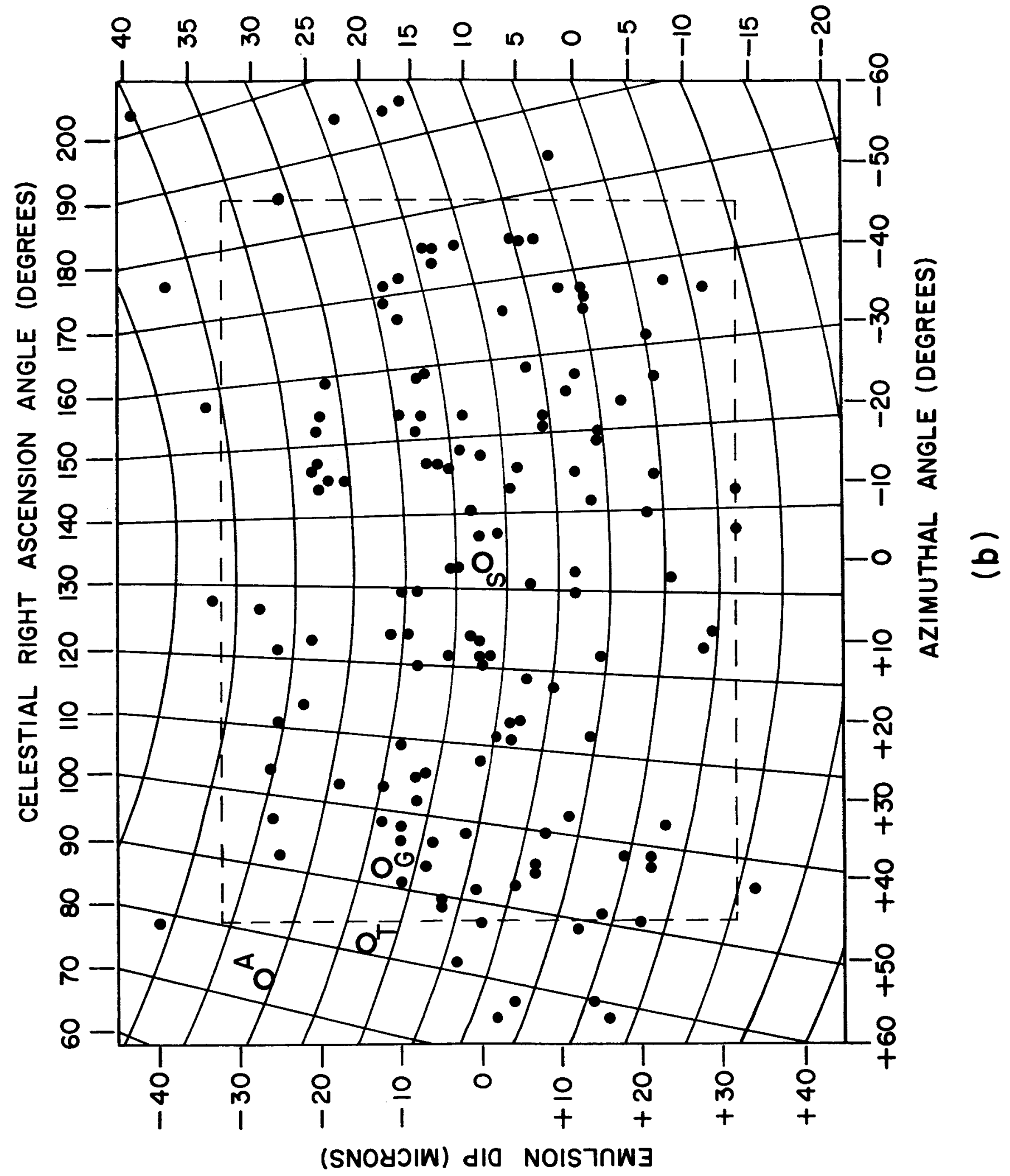

FIGURE 4（b) 


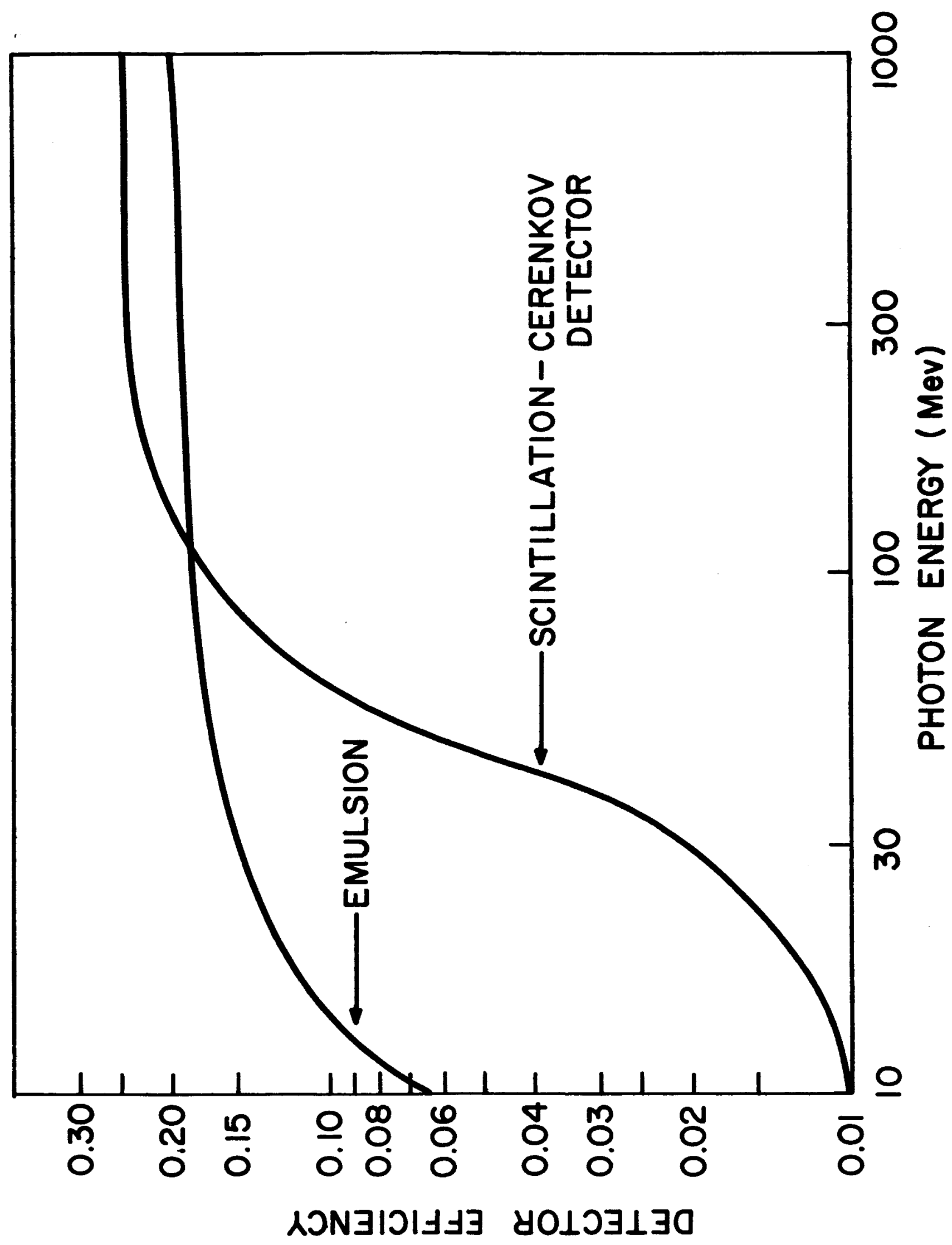

FIGURE 5 